\title{
Context and Auditory Fear are Differentially Regulated by HDAC3 Activity in the Lateral and Basal Subnuclei of the Amygdala
}

\author{
Janine L Kwapis',2, Yasaman Alaghband', Alberto J López', André O White ${ }^{3}$, Rianne R Campbell', \\ Richard T Dang', Diane Rhee', Ashley V Tran', Allison E Carl', Dina P Matheos' and Marcelo A Wood*, I,2 \\ 'Department of Neurobiology and Behavior, Center for the Neurobiology of Learning and Memory, University of California, Irvine, California, USA; \\ ${ }^{2}$ Institute for Memory Impairments and Neurological Disorders, University of California, Irvine, California, USA; ${ }^{3}$ Department of Biological Sciences, \\ Mount Holyoke College, South Hadley, Massachusetts, USA
}

\begin{abstract}
Histone acetylation is a fundamental epigenetic mechanism that is dynamically regulated during memory formation. Histone acetyltransferases (HATs) and histone deacetylases (HDACs) compete to modulate histone acetylation, allowing for rapid changes in acetylation in response to a learning event. HDACs are known to be powerful negative regulators of memory formation, but it is not clear whether this function depends on HDAC enzymatic activity per se. Here, we tested whether the enzymatic activity of an individual Class I HDAC, HDAC3, has a role in fear memory formation in subregions of the hippocampus and amygdala. We found that fear conditioning drove expression of the immediate early genes cFos and Nr4a2 in the hippocampus, which coincided with reduced HDAC3 occupancy at these promoters. Using a dominant-negative, deacetylase-dead point mutant virus (AAV-HDAC3(Y298H)-v5), we found that selectively blocking HDAC3 deacetylase activity in either the dorsal hippocampus or basal nucleus of the amygdala enhanced context fear without affecting tone fear. Blocking HDAC3 activity in the lateral nucleus of the amygdala, on the other hand, enhanced tone, but not context fear memory. These results show for the first time that the enzymatic activity of HDAC3 functions to negatively regulate fear memory formation. Further, HDAC3 activity regulates different aspects of fear memory in the basal and lateral subregions of the amygdala. Thus, the deacetylase activity of HDAC3 is a powerful negative regulator of fear memory formation in multiple subregions of the fear circuit. Neuropsychopharmacology (2017) 42, 1284-1294; doi:I0.1038/npp.2016.274; published online 4 January 2017
\end{abstract}

\section{INTRODUCTION}

For decades, researchers have generally agreed that gene expression is a key requirement for long-term memory formation (Alberini, 2009), but only recently were histone modification mechanisms implicated in this process (Barrett and Wood, 2008; Jarome et al, 2014; Kwapis and Wood, 2014; Maddox et al, 2013a). Epigenetics, broadly defined, includes mechanisms that change gene expression through histone modifications, rather than altering the DNA sequence (Allis et al, 2007). These mechanisms are particularly powerful modulators of memory formation, as they can produce relatively persistent changes at the cellular level that may underlie long-lasting behavioral changes.

Histone acetylation is a fundamental chromatin regulatory mechanism that is dynamically controlled during learning (Levenson et al, 2004; Maddox et al, 2013b; Mahan et al,

* Correspondence: Professor MA Wood, Department of Neurobiology and Behavior, Center for the Neurobiology of Learning and Memory, 2205 McGaugh Hall, Irvine, CA 92697, USA, Tel: +949 824 6I I4, Fax: +949824 6633, E-mail: mwood@uci.edu

Received 18 August 2016; revised 4 November 2016; accepted 2 December 2016; accepted article preview online 7 December 2016
2012; Miller et al, 2008) and critically involved in long-term memory formation (Barrett et al, 2011; Bieszczad et al, 2015; Bredy and Barad, 2008; Guan et al, 2009; Maddox et al, 2013b; McQuown et al, 2011a; Vecsey et al, 2007; Wood et al, 2005). Histone acetylation is modulated through two competing classes of enzymes: HATs and HDACs. Histone acetyltransferases (HATs) add acetyl groups to histone tails, generally promoting a permissive chromatin structure that facilitates gene expression. Histone deacetylases (HDACs) remove acetyl groups, typically promoting a closed chromatin structure that restricts gene expression. The most highly expressed Class I HDAC in the brain, HDAC3, is a powerful negative regulator of memory formation (Malvaez et al, 2013; McQuown et al, 2011a; Rogge et al, 2013). Previous studies have demonstrated that genetic deletion (McQuown et al, 2011a; Rogge et al, 2013) or pharmacological disruption (Bieszczad et al, 2015; Malvaez et al, 2013; Rogge et al, 2013) of HDAC3 transforms a subthreshold learning event into one that generates robust and persistent long-term memory. These results suggest that HDAC3 normally limits memory formation and may also regulate memory strength and persistence. To date, no study has tested whether the deacetylase activity of HDAC3 is specifically necessary for 
its ability to regulate memory formation. This is a key question, considering that HDAC3-mediated gene repression in other tissues does not necessarily require the enzymatic activity of HDAC3 (Sun et al, 2013). Further, another HDAC, HDAC4, can modulate memory independent of its deacetylase domain (Lahm et al, 2007; Sando et al, 2012). Thus, it is important to determine whether the enzymatic activity of HDAC3 is required for memory acquisition.

It is also unclear whether HDAC3 has a role in regulating the persistent, robust aversive associations that underlie fear memory. Studies have demonstrated that general histone acetylation in both the amygdala and hippocampus is critical for fear memory formation. Broadly inhibiting HATs in either structure around the time of learning impairs fear memory (Barrett et al, 2011; Maddox et al, 2013b; Maddox et al, 2013c). Pharmacological broad-spectrum HDAC inhibition in either the dorsal hippocampus (DH; Vecsey et al, 2007) or amygdala (Monsey et al, 2011; Yeh et al, 2004) has the opposite effect, enhancing fear memory. To date, no one has tested the roles of individual HDACs directly in the amygdala or hippocampus during fear memory formation. In this study, we examined the role of HDAC3 deacetylase activity in the $\mathrm{DH}$ and subregions of the amygdala during auditory and context fear memory formation.

\section{MATERIALS AND METHODS}

\section{Subjects}

Subjects were 133 adult male C57BL/6 J mice (8-weeks-old; Jackson Laboratory). The mice were housed, fed, and handled as described in the Supplementary Methods. All the procedures were approved by the University of California, Irvine's Institutional Animal Care and Use Committee and were in compliance with the National Institutes of Health guidelines.

\section{Surgery}

The animals were injected with either AAV-HDAC3 (Y298H)-v5 or AAV-EV (empty vector). For DH infusions, $1 \mu \mathrm{l}$ of virus was infused bilaterally. For infusions into the BA or LA, $0.5 \mu \mathrm{l}$ was infused into each hemisphere. Immunofluorescence was used to confirm expression of AAVHDAC3(Y298H)-v5.

\section{Quantitative RT-PCR}

Quantitative real-time RT-PCR was performed to examine learning-induced gene expression or to verify viral expression following infusions as previously described (Lopez et al, 2016; White et al, 2016). Specific primer and probe sequences are listed in the Supplementary Methods.

\section{Chromatin Immunoprecipitation}

ChIP was performed as described previously (Malvaez et al, 2013; Rogge et al, 2013), based on the protocol from the Millipore ChIP kit (Supplementary Methods). Sheared chromatin was immunoprecipitated with an HDAC3 antibody (Millipore) or anti-mouse IgG (negative control,
Millipore), and Fos and Nr4a2 promoter enrichment was measured using qPCR.

\section{Fear Conditioning}

Fear conditioning and context tests were conducted in Context A, whereas auditory tests were conducted in Context B. The animals were trained with either contextonly fear conditioning (Figure 1, Supplementary Figure S1) or cued fear conditioning (Figures 2-5, Supplementary Figures S2 and S4) and tested to the context and/or the tone the following day. For molecular work, the mice were killed 30 or 60 min after training.

\section{Statistical Analysis}

Freezing behavior was used to measure conditional fear. For context tests, the average freezing during the first 3 min was used for statistical analysis (Supplementary Methods). For tone tests, we used average freezing during the CS period for statistical analyses. Group differences were analyzed with Student's $t$-tests or mixed-model ANOVAs and Sidak's multiple comparison post hoc tests and an $\alpha$-value of 0.05 was required for significance.

\section{RESULTS}

\section{Fear Conditioning Drives HDAC3-Mediated Gene Expression in the Hippocampus}

We first confirmed that fear conditioning triggers HDAC3mediated gene expression in the hippocampus. The mice were trained with context fear conditioning and killed 30 or $60 \mathrm{~min}$ after the end of the conditioning session (Figure 1a; $n=9-10$ per group). Homecage (HC) mice were treated identically except that they received no training session and were killed between behavior groups. We measured hippocampal mRNA expression of two immediate early genes known to be direct targets regulated by HDAC3: cFos and Nr4a2 (Malvaez et al, 2013; McQuown et al, 2011a; Rogge et al, 2013). We first measured cFos expression, often used as a marker of activity following learning (Kubik et al, 2007; Lehner et al, 2009; Milanovic et al, 1998). cFos was significantly increased in the $\mathrm{DH}$ after fear conditioning (one-way ANOVA, $\mathrm{F}_{(2,26)}=23.3, p<0.001$ ), with significantly higher expression at $30 \mathrm{~min}$ (Sidak post hoc, $p<0.001$ ) and $60 \mathrm{~min} \quad(p<0.001)$ relative to homecage controls (Figure 1b). We also measured $N r 4 a 2$, which is both CREB-dependent (Conkright et al, 2003; McNulty et al, 2012; Vecsey et al, 2007) and regulated by HDAC3 (Rogge et al, 2013). Nr4a2 was significantly increased after acquisition $\left(\mathrm{F}_{(2,26)}=7.8, p<0.01\right)$, with significantly higher expression $30 \mathrm{~min}$ after conditioning $(p<0.01)$ and $60 \mathrm{~min}$ after learning (Figure 1c; $p<0.01$ ).

In the ventral hippocampus, a similar pattern emerged (Supplementary Figure S1). We observed significantly higher cFos expression in the ventral hippocampus after conditioning $\left(\mathrm{F}_{(2,12)}=23.5, p<0.05 ; n=5\right.$ per group), both at $30 \mathrm{~min}$ $(p<0.05)$ and $60 \mathrm{~min}(p<0.05)$ compared with controls (Supplementary Figure S1A). Although Nr4a2 mRNA expression was not significantly increased $\left(\mathrm{F}_{(2,11)}=1.24\right.$, $p>0.05, n=4-5$ per group), we did observe a slight, 
a

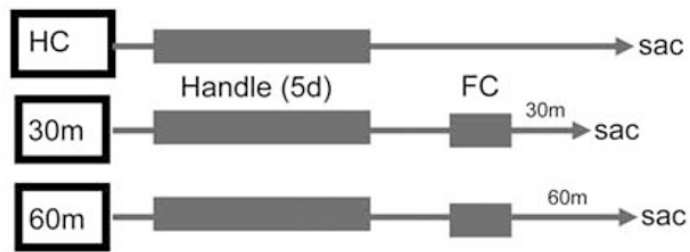

b

cFos mRNA

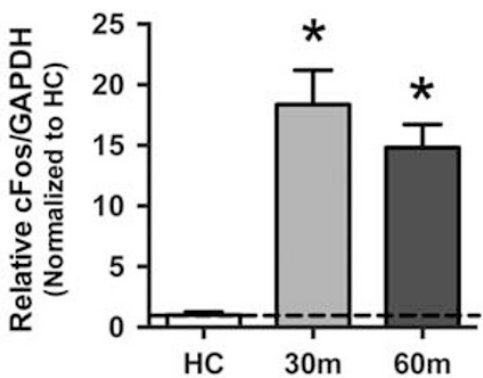

d

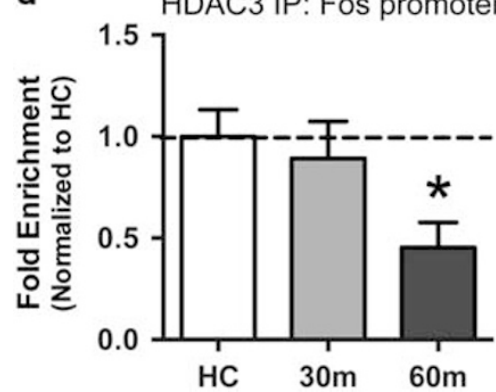

c

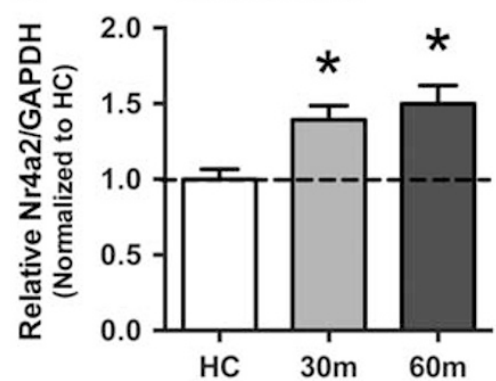

e

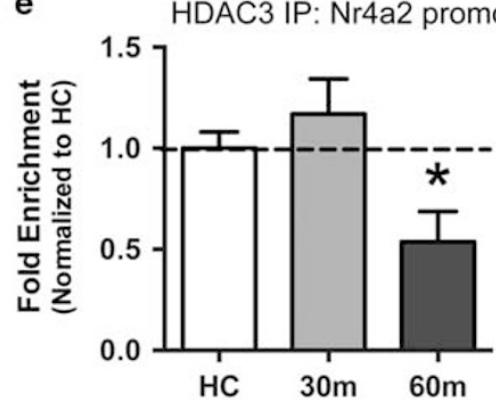

Figure I Fear conditioning drives cFos and Nr4a2 mRNA and reduces HDAC3 occupancy at these gene promoters in the dorsal hippocampus. (a) Experimental timeline. (b) RT-qPCR revealed significant increases in cFos mRNA in the dorsal hippocampus (DH) 30 and 60 min after fear conditioning. (c) Nr4a2 mRNA also increases in DH at 30 and 60 min after learning. For DH mRNA, n=9-10/group. (d) ChIP was performed with anti-HDAC3 followed by qPCR to identify binding to the cFos (d) or Nr4a2 (e) promoters. HDAC3 occupancy at both promoter regions was significantly reduced at 60 min after training compared with home cage controls. For ChIP, $n=7-10 /$ group. * $p<0.05$ compared with HC control.
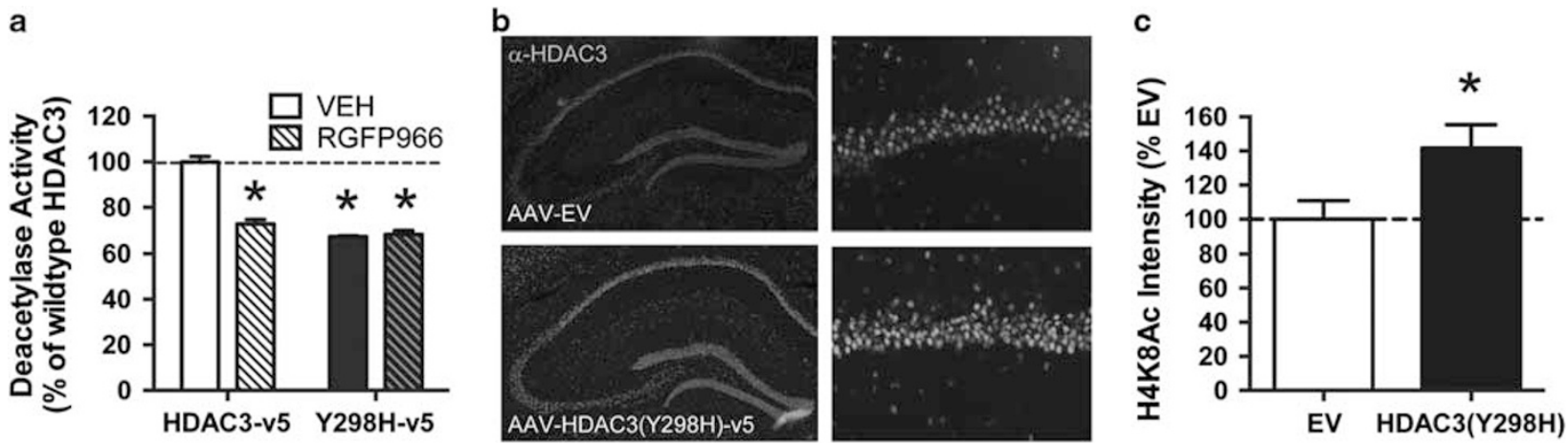

Figure 2 The dominant-negative point mutant HDAC3(Y298H)-v5 blocks deacetylation in vitro and augments histone acetylation in vivo in the dorsal hippocampus. (a) HDAC3Y298H reduced deacetylase activity in vitro as effectively as the HDAC3-specific inhibitor RGFP966. (b, c) Mice infused with empty vector (AAV-EV) or point mutant (AAV-HDAC3(Y298H)-v5) viruses were trained in fear conditioning and histone 4, lysine 8 acetylation (H4K8Ac) was measured 30 min later with immunofluorescence. H4K8Ac was significantly increased in the DH of mice infused with AAV-HDAC3(Y298H)-v5. For activity assay, $n=3$ /group. For H4K8Ac immunofluorescence, $n=9$ /group. $* p<0.05$ compared with VEH or EV control.

nonsignificant increase in expression after learning (Supplementary Figure S1B). Together, these results demonstrate that fear conditioning activates gene expression throughout the hippocampus.

To determine whether this learning-induced gene expression in the DH is mediated through HDAC3, we measured
HDAC3 occupancy at the promoter regions of $c F o s$ and Nr4a2 using ChIP. HDAC3 was enriched at the cFos promoter in both homecage controls $(n=10)$ and at 30 min $(n=7)$ following fear conditioning but HDAC3 occupancy at the cFos promoter was significantly reduced at $60 \mathrm{~min}$ compared with the homecage group (Figure 1d; 
one-way ANOVA: $\mathrm{F}_{(2,22)}=4.09 p<0.05$; Sidak post hoc, HC vs $30 \mathrm{~min}, p>0.05$; HC vs $60 \mathrm{~min}, p<0.05)$. This indicates that HDAC3 disassociates from the cFos promoter $30 \mathrm{~min}$ after training. We observed a similar pattern for $\mathrm{Nr} 4 a 2$ (Figure 1e; $\mathrm{F}_{(2,21)}=5.8, p<0.01$ ), with reduced occupancy of HDAC3 at the $N r 4 a 2$ promoter in the 60 min group compared with HC controls $(p<0.05 ; \mathrm{HC} n=8,30 \mathrm{~min}$ $n=7)$ but no significant reduction at $30 \mathrm{~min}$ post training $(p>0.05 ; n=7)$. This suggests that HDAC3 occupies both the $c$ Fos and Nr4a2 promoter regions at rest and shortly after fear conditioning, but is dissociated from the chromatin around an hour after learning. Together, these experiments demonstrate that HDAC3-regulated gene expression is induced in the hippocampus following aversive memory formation.

\section{Disrupting HDAC3 Activity with AAV-HDAC3(Y298H)- v5 Blocks Deacetylase Activity and Promotes Histone Acetylation at $\mathrm{H} 4 \mathrm{~K} 8$}

HDAC3 is known to negatively regulate some forms of learning (Bieszczad et al, 2015; Malvaez et al, 2013; McQuown et al, 2011a; Rogge et al, 2013), but it is unclear whether the deacetylase activity of HDAC3 is specifically required for this function. To test whether HDAC3 activity negatively regulates fear memory formation, we developed a dominant-negative point mutant virus (AAV2.1-HDAC3 (Y298H)-v5) to selectively disrupt HDAC3 activity. Substituting a histidine in place of the tyrosine abolishes the enzymatic activity of HDAC3 without affecting proteinprotein interactions (Lahm et al, 2007; Sun et al, 2013). To confirm that HDAC3(Y298H) mutation effectively blocks HDAC3-mediated deacetylation, we performed an in vitro HDAC3 deacetylase activity assay using purified HDAC3 (Y298H)-v5 or wild-type HDAC3-v5 protein. We found that HDAC3(Y298H) reduced deacetylase activity to the same extent as the HDAC3-specific inhibitor RGFP966 (Bieszczad et al, 2015; Figure 2a). A two-way ANOVA revealed a significant effect for virus $\left(\mathrm{F}_{(1,9)}=144.8, p<0.05\right)$, drug $\left(\mathrm{F}_{(1,9)}=74.55, p<0.05\right)$, and a significant interaction $\left(F_{(1,9)}=85.72, p<0.05\right)$. Follow-up Sidak's tests found that RGFP966 significantly reduced activity in the wild-type HDAC3 group $(p<0.05)$ but did not further decrease HDAC3 activity in the HDAC3 $(\mathrm{Y} 298 \mathrm{H})$ group $(p>0.05)$. Thus, the HDAC3(Y298H) point mutant is an appropriate tool to block HDAC3 deacetylase activity.

Disrupting HDAC3 typically results in increased histone acetylation, specifically at lysine 8 on $\mathrm{H} 4$ (Malvaez et al, 2013; McQuown et al, 2011a; Rogge et al, 2013). To confirm that AAV-HDAC3(Y298H)-v5 augments histone acetylation, we bilaterally infused the point mutant virus or an empty vector (AAV-EV) virus into the $\mathrm{DH}$ and examined $\mathrm{H} 4 \mathrm{~K} 8$ acetylation in the DH $30 \mathrm{~min}$ after acquisition (Figure 2b; $n=9$ /group). The mice infused with AAV-HDAC3(Y298H) had significantly higher $\mathrm{H} 4 \mathrm{~K} 8$ acetylation levels than EV control mice (Figure 2c; $t_{(16)}=2.838, p<0.05$ ). Blocking the deacetylase activity of HDAC3 with AAV-HDAC3 (Y298H)-v5 therefore augmented learning-induced histone acetylation.
Blocking HDAC3 Activity in the Dorsal Hippocampus with AAV-HDAC3(Y298H)-v5 Enhances Context, But Not Auditory Fear Conditioning

Our initial experiments suggest that HDAC3-regulated gene expression is induced in the $\mathrm{DH}$ during fear memory formation (Figure 1). To test whether HDAC3 activity specifically constrains the formation of fear memory in the $\mathrm{DH}$, mice were given bilateral infusions of either the point mutant virus (AAV-HDAC3(Y298H)-v5) or control (AAV-EV). Two weeks later (allowing for optimal expression (Barrett et al, 2011; McQuown et al, 2011a; Rogge et al, 2013)), mice were trained with a single toneshock pairing (Figure 3a). The following day, the mice were tested serially to the context and auditory CS to determine whether disrupting HDAC3 activity affected long-term memory.

To confirm that our viral infusion appropriately targeted the $\mathrm{DH}$, we measured immunoreactivity to the V5 epitope on the point mutant virus. We observed efficient transduction of AAV-HDAC3(Y298H)-v5 throughout areas CA1 and CA3 of the DH of all AAV-HDAC3(Y298H)-v5 animals, with no V5 staining observed in animals infused with the AAV-EV control virus (Figure $3 \mathrm{~b}$ and $c$; $t_{(17)}=3.609, p<0.05, n=9-10$ per group). We also measured wild-type $H d a c 3$ and mutated Hdac3(Y298H)-v5 mRNA expression in DH punches taken from this region using RT-qPCR (Supplementary Figure S2). Primers against both the v5-containing HDAC3(Y298H) transcript and the endogenous Hdac3 transcript (which recognizes both endogenous $\mathrm{Hdac} 3$ and mutated Hdac3 (Y298H)-v5 mRNA) confirmed significantly higher levels of both transcripts in mice infused with AAV-HDAC3 (Y298H)-v5. Finally, we assessed the spread of V5 through the dorsal hippocampus by measuring V5 immunoreactivity throughout the hippocampus. The virus expressed throughout CA1 and CA3 of the dorsal hippocampus with very little expression observed in the dentate gyrus and no observable expression in the ventral hippocampus or elsewhere in the brain (Supplementary Figure S3). Together, these results demonstrate that our infusion of AAV-HDAC3(Y298H) correctly targeted the $\mathrm{DH}$ and drove expression of both mRNA and protein encoding HDAC3(Y298H)-v5.

We then tested whether disrupting HDAC3 activity in the DH with AAV-HDAC3(Y298H)-v5 would affect context or auditory fear conditioning. All the animals showed normal acquisition on day 1 (Supplementary Figure S4A) with no group differences in freezing observed (one-way ANOVA: $\left.\mathrm{F}_{(2,27)}=2.75, p>0.05\right)$. The following day, the animals were tested to both the context and auditory CS (Figure $3 \mathrm{~d}-\mathrm{g}$ ). Infusion of AAV-HDAC3(Y298H)-v5 into the DH enhanced context fear without affecting freezing to the auditory CS. During the context test, AAV-HDAC3(Y298H)-v5 mice froze significantly more than AAV-EV mice (Figure 3d; $\left.t_{(17)}=5.74, p<0.05\right)$. A minute-by-minute analysis revealed that AAV-HDAC3(Y298H) mice showed initially high levels of freezing that rapidly diminished by the end of the session (Figure 3e). A mixed-model ANOVA revealed a significant effect of minute $\left(\mathrm{F}_{(4,68)}=9.83, p<0.001\right)$, virus $\left(\mathrm{F}_{(1,17)}=31.12, p<0.05\right)$ and a significant minute $\times$ virus interaction $\left(\mathrm{F}_{(4,68)}=2.56, p<0.05\right)$. Follow-up Sidak's tests revealed that AAV-HDAC3(Y298H) mice froze significantly 


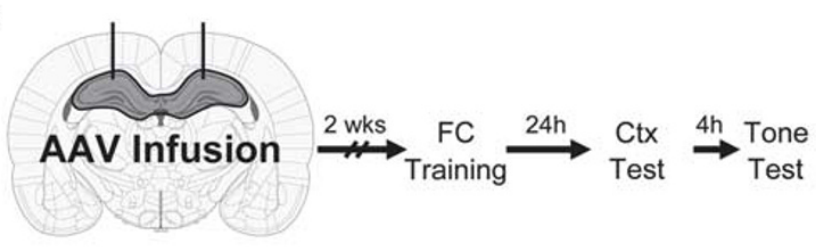

b
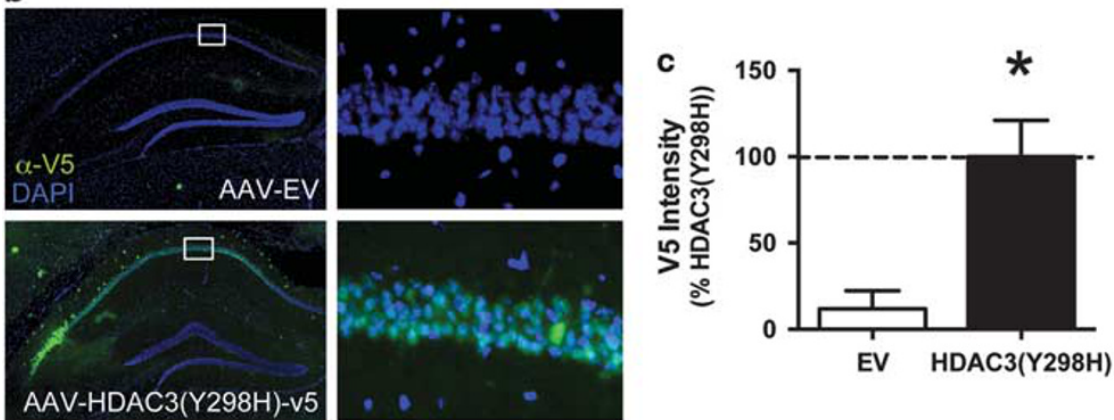

d
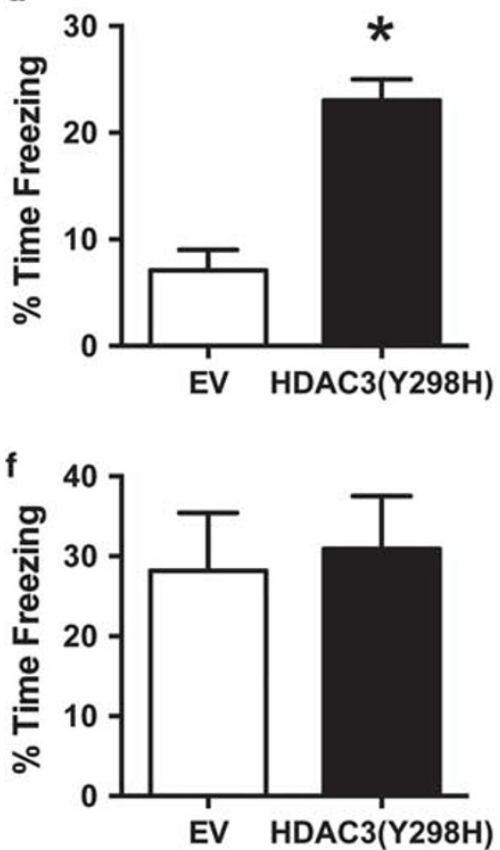

e
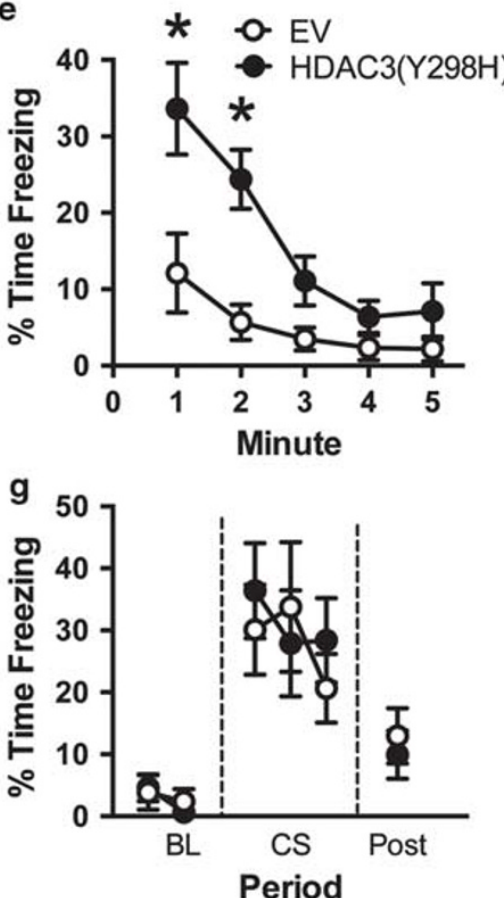

Figure 3 Blocking HDAC3 activity in the DH enhances context fear without affecting auditory CS fear. (a) Experimental design. Two weeks after infusion of AAV-EV or AAV-HDAC3(Y298H)-v5, the mice were trained in fear conditioning. The following day, the mice were tested serially to both the context and tone. After the completion of behavior, expression of the virus was confirmed. (b) Representative immunofluorescence images showing expression of the $V 5$ epitope tag in the dorsal hippocampus (green). Nuclei are counterstained with DAPI (blue). No V5 expression was observed in mice infused with AAV-EV (top), whereas strong V5 expression was observed in CAI and CA3 of mice infused with AAV-HDAC3(Y298H)-v5 (bottom). (c) Quantification of this staining confirmed significantly higher expression of $v 5$ in HDAC3(Y298H)-v5 animals compared with EV controls. For AAV-EV group, $n=9$. For HDAC3 $(\mathrm{Y} 298 \mathrm{H})$-v5 group, $n=10$. ${ }^{*} p<0.05$ compared with EV control. (d, e) Freezing during the context test. (d) Mice given AAV-HDAC3(Y298H)-v5 showed significantly higher freezing during the first 3 min of the context test than AAV-EV mice. (e) Minute-by-minute analysis of this session confirmed that AAVHDAC3(Y298H)-v5 mice showed significantly higher freezing during minutes I and 2 of the context test. (f, g) Freezing during the tone test. (f) Both groups froze at similar levels during the tone presentation. (g) Similarly, a minute-by-minute analysis of the tone test session revealed no group differences. For EV, $n=9$. For HDAC3 $(Y 298 \mathrm{H}), n=10 . * 2<0.05$ compared with EV.

more than EV mice during minutes $1(p<0.05)$ and 2 $(p<0.05)$.

In contrast, we observed no differences in freezing during the tone test. Both groups showed similar freezing levels during the tone presentation (Figure 3f; $t_{(17)}=0.784$, $p>0.05$ ). A minute-by-minute analysis (Figure $3 \mathrm{~g}$ ) also failed to reveal any effects of virus within the tone test. Together, these data demonstrate that infusion of AAVHDAC3(Y298H)-v5 into the dorsal hippocampus enhances context fear without affecting fear to the auditory CS, consistent with the well-documented role of the $\mathrm{DH}$ in supporting context, but not auditory fear conditioning. 
a

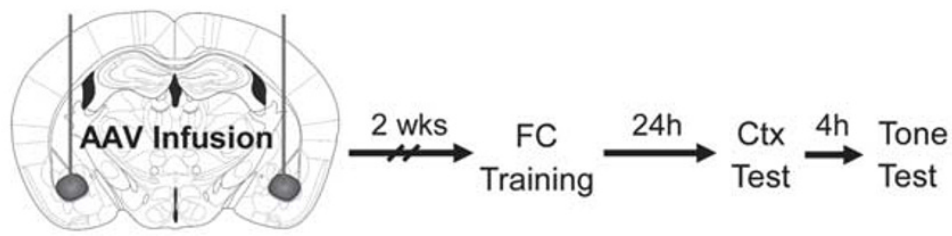

b

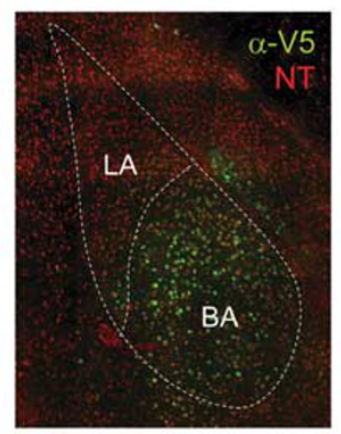

d
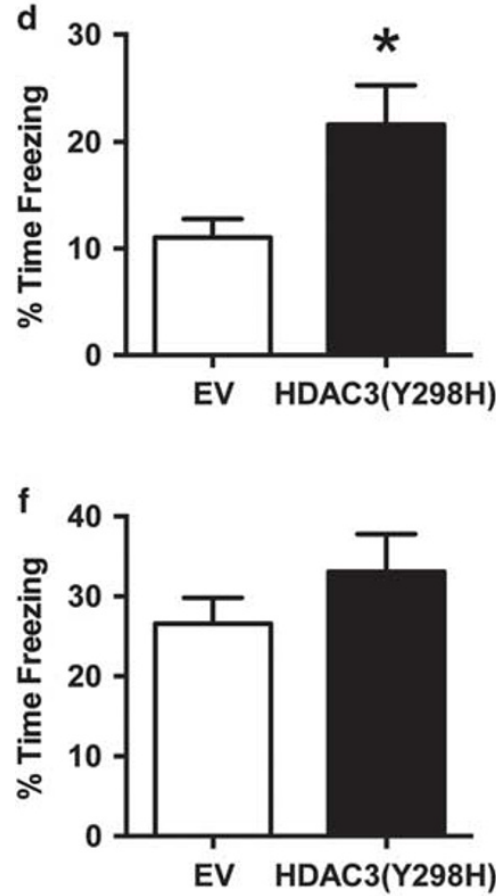

c

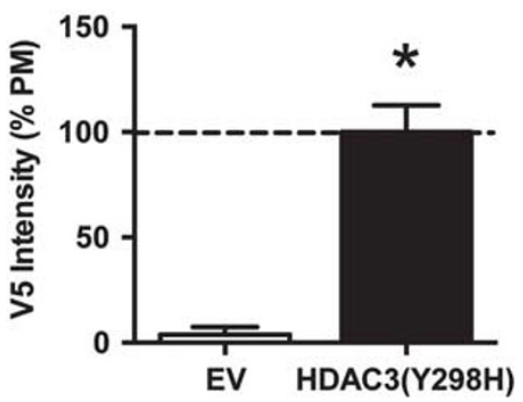

e
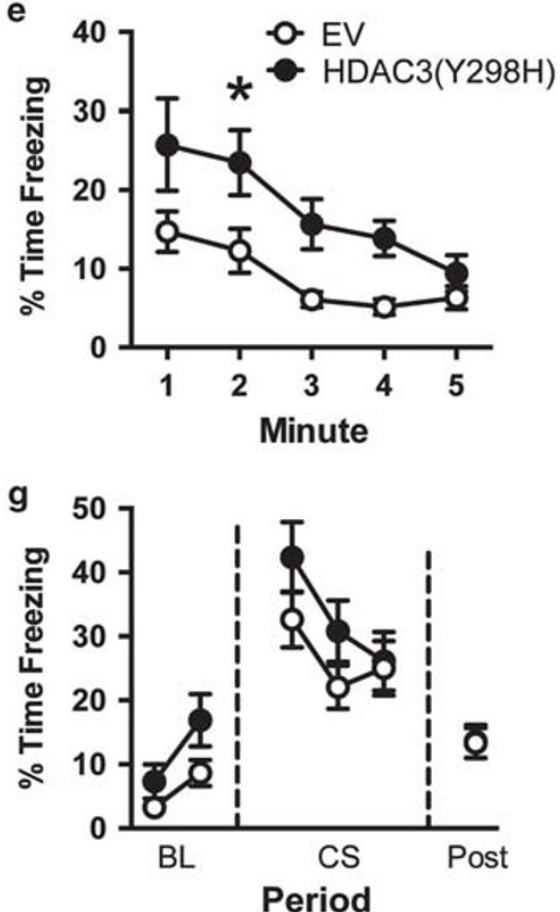

Figure 4 Blocking HDAC3 activity in the basal amygdala with HDAC3(Y298H)-v5 enhances context fear without affecting auditory CS fear. (a) Experimental design. (b) Representative immunofluorescence image showing expression of the $\mathrm{V} 5$ epitope tag (green) in the basal amygdala (BA) after infusion of AAV-HDAC3(Y298H)-v5. Neurons are counterstained with NeuroTrace (red), a fluorescent Nissl stain. V5 expression was largely confined to the basal nucleus of the amygdala. (c) Quantification of immunostaining confirmed significantly higher expression of v5 in HDAC3(Y298H)-v5 mice compared with EV controls. (d, e) Freezing during the context test. (d) Mice given AAV-HDAC3(Y298H)-v5 showed significantly higher freezing during the first 3 min of the context test than AAV-EV mice. (e) Minute-by-minute analysis of this session determined that AAV-HDAC3(Y298H)-v5 mice showed significantly higher freezing during minute 2 of the context test. $(f, g)$ Freezing during the tone test. $(f)$ Both groups froze at similar levels during the tone presentation. (g) Minuteby-minute analysis of this test session similarly failed to reveal any group differences. $n=15 /$ group. $* p<0.05$ compared with $E V$.

Disrupting HDAC3 Activity in the Basal Amygdala Also Enhances Context, But Not Auditory Fear Conditioning

To further investigate the role of HDAC3 activity in fear memory formation, we also tested the effects of AAVHDAC3(Y298H)-v5 in the amygdala. We initially tested the virus in the basal nucleus of the amygdala (BA), which receives input from the $\mathrm{DH}$ and has a key role in context fear conditioning (Canteras and Swanson, 1992; LeDoux, 2000; Yaniv et al, 2004). We bilaterally infused AAV-HDAC3 (Y298H)-v5 or AAV-EV into the BA before conditioning mice (Figure 4a) as previously described (Figure 3).

We confirmed the location of our infusions by measuring immunoreactivity to the V5 epitope of the point mutant virus. As shown in Figure 4b, HDAC3(Y298H)-v5 was expressed throughout the BA with only minor expression 
a

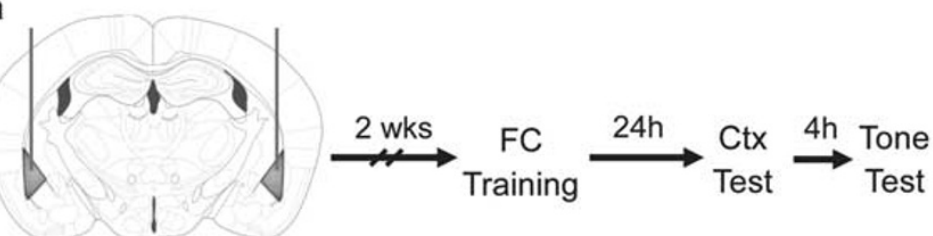

b

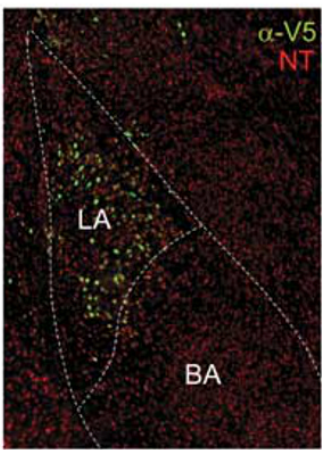

d
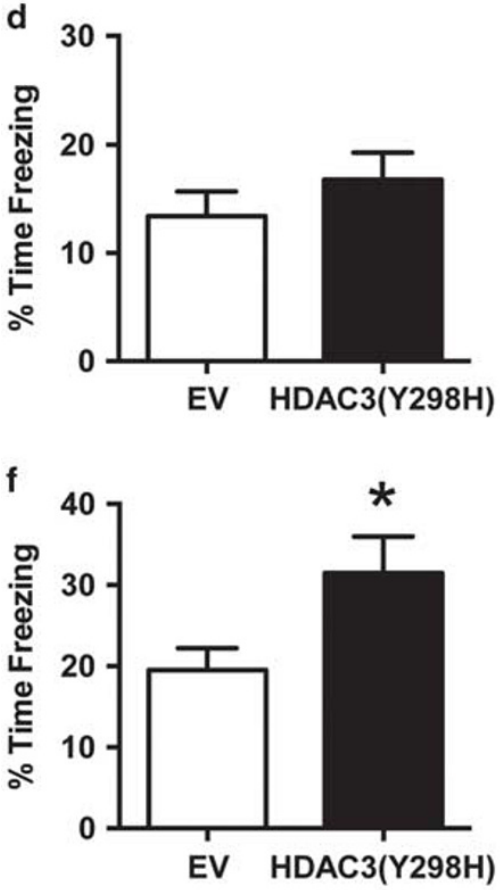

C

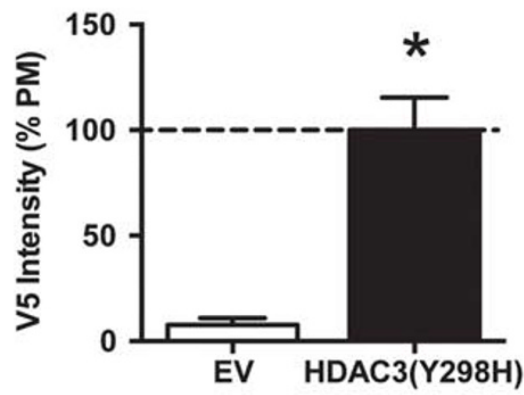

e
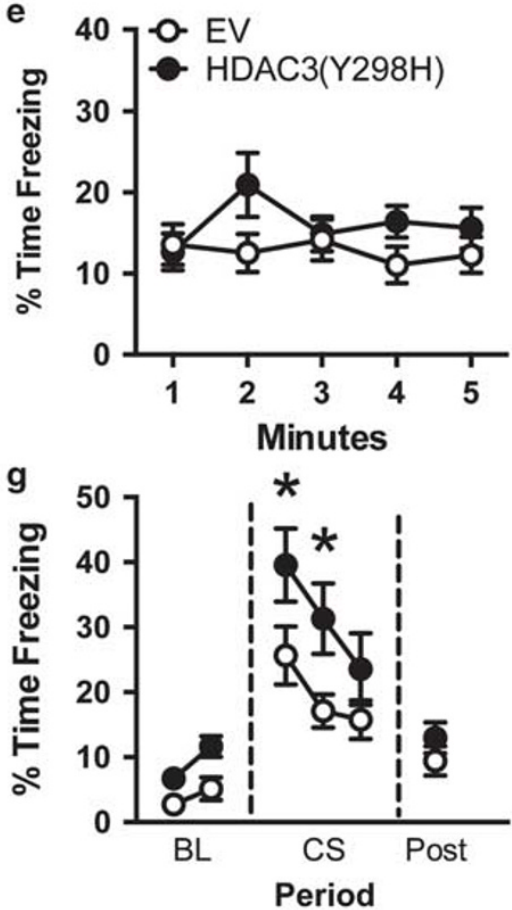

Figure 5 Blocking HDAC3 activity in the lateral amygdala with HDAC3(Y298H)-v5 enhances fear to the tone CS without affecting context fear. (a) Experimental design. (b) Representative immunofluorescence image showing expression of the V5 epitope tag (green) in the lateral amygdala (LA) after infusion of AAV-HDAC3(Y298H)-v5. Neurons are counterstained with NeuroTrace (red), a fluorescent Nissl stain. V5 expression was largely confined to the lateral nucleus of the amygdala. (c) Quantification of immunostaining confirmed that v5 expression was higher in HDAC3(Y298H)-v5 mice compared with EV controls. (d, e) Freezing during the context test. (d) Both groups froze at similar levels during the context test. (e) Minute-by-minute analysis of the context tests similarly failed to reveal any group differences. (f, g) Freezing during the tone test. (f) Mice given AAV-HDAC3(Y298H)-v5 showed significantly higher freezing during the tone presentation than mice given AAV-EV. (g) Minute-by-minute analysis of the tone test revealed that mice froze at similar levels during the baseline period (BL), but mice given AAV-HDAC3(Y298H)-v5 showed significantly higher freezing than mice given AAV-EV during the first 2 min of the tone presentation (CS; minutes 3 and 4). No group differences were observed in the 30 s period after the CS offset (post). For EV, $n=9$. For HDAC3 $(\mathrm{Y} 298 \mathrm{H}), n=10 . * p<0.05$ compared with EV.

observed in the lateral nucleus or central nucleus of the amygdala. Quantification of this staining confirmed that brains infused with AAV-HDAC3(Y298H)-v5 expressed significantly more $\mathrm{V} 5$ protein in the BA than brains infused with AAV-EV (Figure $4 c ; t_{(28)}=7.265, p<0.05 ; n=15$ per group). Our infusion of AAV-HDAC(Y298H)-v5 therefore appropriately targeted the BA.

To determine the effects of this infusion on fear memory, we trained the animals as before, and tested both context and auditory fear the following day (Figure $4 \mathrm{~d}-\mathrm{g}$ ). All the animals 
showed normal acquisition on day 1 (Supplementary Figure S4B) and no group differences were observed in freezing levels during the training session $\left(t_{(28)}=0.353\right.$, $p>0.05)$. During testing the following day, we found that intra-BA infusion of AAV-HDAC3(Y298H)-v5 enhanced context fear without significantly affecting fear to the auditory CS, similar to the effects we observed in the $\mathrm{DH}$. During the context test, HDAC3(Y298H)-v5 mice showed significantly higher freezing than mice given AAV-EV (Figure $4 \mathrm{~d} ; t_{(28)},=2.642, p<0.05$ ). A minute-by-minute analysis (Figure 4e) showed that freezing reduced over the 5 -min test session (main effect of minute $\left(\mathrm{F}_{(4,112)}=11.30\right.$, $p<0.05)$ ) with a significant effect of virus $\left(\mathrm{F}_{(1,28)}=7.962\right.$, $p<0.05)$, but no significant interaction $\left(\mathrm{F}_{(4,112)}=1.038\right.$, $p>0.05))$. Follow-up Sidak's tests found that AAV-HDAC3 $(\mathrm{Y} 298 \mathrm{H})-\mathrm{v} 5$ mice froze significantly more than AAV-EV mice during minute $2(p<0.05)$. Thus, mice infused with AAV-HDAC3(Y298H)-v5 froze significantly more to the context, with both groups showing a reduction in freezing over the course of the context test.

During the tone test (Figure $4 \mathrm{f}-\mathrm{g}$ ), we observed no difference in freezing between groups (Figure 4f; $\left.t_{(28)}=1.146, \quad p>0.05\right)$. A minute-by-minute analysis (Figure 4g) failed to reveal any effects of virus within any period of the tone test session. These results together suggest that AAV-HDAC3(Y298H) infusion into the BA enhances context fear without affecting tone CS fear. Importantly, every animal included in the behavioral data presented was shown to have proper viral expression isolated to the BA (see Figure 4b).

\section{Blocking HDAC3 Activity in the Lateral Nucleus of the Amygdala with AAV-HDAC3(Y298H)-v5 Enhances Auditory, But Not Context Fear Conditioning}

Our results suggest that HDAC3 activity is a key negative regulator of context fear, but it is unclear whether fear to the auditory CS is also regulated by HDAC3 activity. Studies examining the role of the histone acetyltransferase CREBbinding protein (CBP) have led to conflicting results whether CBP and histone acetylation are necessary for auditory fear memory (Kwapis and Wood, 2014). To determine whether HDAC3-mediated deacetylation negatively regulates auditory fear conditioning, we next examined at the effects of HDAC3(Y298H) in the lateral nucleus of the amygdala (LA), a key site of plasticity involved in auditory fear conditioning (Amorapanth et al, 2000; Goosens and Maren, 2001; Schafe et al, 2005). To test this, we bilaterally infused AAV-HDAC3 (Y298H)-v5 or AAV-EV into the LA before fear acquisition (Figure 5a).

To confirm our infusion sites, we again measured V5 immunoreactivity and confirmed that HDAC3(Y298H)-v5 was appropriately expressed in the LA with little expression in either the BA or central nucleus of the amygdala (Figure 5b). Quantification confirmed that brains infused with AAV-HDAC3(Y298H)-v5 expressed more V5 protein in the LA than brains infused with AAV-EV (Figure 5c; $t_{(17)}=5.737, \quad p<0.05 ; n=9-10$ per group). Thus, our infusion of AAV-HDAC3(Y298H)-v5 into the LA was targeted appropriately.

We then tested whether disrupting HDAC3 activity in the LA with HDAC3(Y298H)-v5 would affect auditory or context fear conditioning. All the animals showed normal acquisition (Supplementary Figure S4C) and no group differences were observed in freezing during the training session $\left(t_{(18)}=0.318, p>0.05\right)$. The test sessions the following day (Figure $5 \mathrm{~d}-\mathrm{g}$ ) revealed that infusion of $\mathrm{AAV}$ HDAC3(Y298H)-v5 into the LA had no effect on context fear but enhanced fear to the discrete auditory CS. For the context test, we observed no difference in freezing between AAV-EV $(n=9)$ and AAV-HDAC3(Y298H) $(n=10)$ mice (Figure $5 \mathrm{~d}$; $\left.t_{(17)}=0.989, \quad p>0.05\right)$. A minute-by-minute analysis (Figure 5e) similarly revealed no effect of virus $\left(\mathrm{F}_{(1,18)}=1.662, p>0.05\right)$, minute $\left(\mathrm{F}_{(4,72)}=0.959, p>0.05\right)$, or virus $\times$ minute interaction $\left(\mathrm{F}_{(4,72)}=1.675, p>0.05\right)$. AAVHDAC3(Y298H) infusion therefore did not enhance context freezing.

During the tone test, mice given intra-LA infusion of AAV-HDAC3(Y298H) showed significantly greater freezing during the tone presentation than mice infused with AAVEV (Figure 5f; $t_{(17)}=2.22, p<0.05$ ). Minute-by-minute analysis of this session (Figure $5 \mathrm{~g}$ ) confirmed significant main effects of both minute $\left(\mathrm{F}_{(5,85)}=25.39, p<0.05\right)$ and virus $\left(\mathrm{F}_{(1,17)}=6.595, p<0.05\right)$ and Sidak's post hoc tests revealed that AAV-HDAC3(Y298H) mice froze significantly more than control mice during minutes $3(p<0.05)$ and 4 $(p<0.05)$, the first $2 \mathrm{~min}$ of the CS presentation. Infusion of AAV-HDAC3 $(\mathrm{Y} 298 \mathrm{H})$ into the lateral nucleus of the amygdala therefore enhanced tone CS fear without affecting freezing to the context. Importantly, every animal included in the behavioral data presented was shown to have proper viral expression isolated to the LA (Figure 5b).

\section{DISCUSSION}

Currently, little is known about the specific roles played by individual HDACs in subregions of the neural circuit supporting fear memory formation. Further, it is unclear whether the deacetylase activity of these HDACs is specifically responsible for negatively regulating memory consolidation. Here, we show for the first time that the deacetylase activity of HDAC3 negatively regulates fear memory formation in the dorsal hippocampus, basal nucleus of the amygdala, and lateral nucleus of the amygdala. We found that context fear conditioning drives $c$ Fos and $\mathrm{Nr} 4 a 2$ mRNA expression in the $\mathrm{DH}$, as well as reduced HDAC3 occupancy at these genes. We also found that selectively blocking HDAC3 activity in the dorsal hippocampus or basal nucleus of the amygdala enhances context fear without affecting fear to the auditory CS. Finally, we found that blocking HDAC3 activity in the lateral nucleus of the amygdala selectively enhanced auditory, but not context fear. Together, these experiments show for the first time that both auditory and context fear memory formation require HDAC3 activity.

Recent studies have demonstrated that HDAC3 is a key negative regulator of memory formation in other tasks, including spatial object location memory (McQuown et al, 2011a), reward-based cocaine conditioned place-preference (CPP; Rogge et al, 2013), and extinction of cocaine CPP (Malvaez et al, 2013). Presumably, these memory enhancements occur because genetic deletions or pharmacological disruptions block the deacetylase activity of HDAC3, which 
ultimately promotes gene expression and memory formation. These effects may arise, however, because these HDAC3 manipulations block HDAC3 interactions with other proteins, preventing the formation of the HDAC3 complex with co-repressors NCoR and SMRT and Class II HDACs 4 and 5 (McQuown and Wood, 2011b; Vogel-Ciernia and Wood, 2012). Indeed, both genetic deletion and pharmacological blockade of HDAC3 also decrease expression and/or localization of HDAC4 (McQuown et al, 2011a), suggesting that these HDAC3 manipulations may disrupt formation of the entire repressor complex. As successful formation of the HDAC3 co-repressor complex is required for deacetylase function (Guenther et al, 2001; Guenther et al, 2000), blocking HDAC3 protein-protein interactions would effectively disrupt deactylation even without any manipulation of HDAC3's deacetylation domain per se. Consistent with this, simply blocking the interaction between HDAC3 and its corepressor NCoR is sufficient to enhance memory (McQuown et al, 2011a). Thus, to determine whether the enzymatic activity of HDAC3 itself has a role in regulating memory, it is crucial that normal HDAC3 protein-protein interactions are preserved. Here, to confirm that a functional HDAC3 deactylase domain is critically important for HDAC3's ability to regulate memory, we used a dominant-negative point mutant virus (AAV-HDAC3(Y298H)-v5) that selectively abrogates the deacetylase activity of HDAC3 without affecting its protein-protein interactions (Lahm et al, 2007; Sun et al, 2013). This is the first demonstration that selectively blocking the deacetylase activity of an individual HDAC is sufficient to enhance memory formation.

Another important finding of this study is that manipulations of the LA and BA have different effects on auditory and context fear. Specifically, we found that infusions of AAVHDAC3(Y298H) targeted to the BA only enhanced context fear whereas infusions targeted to the LA selectively enhanced tone fear. Previous studies manipulating histone acetylation have not consistently found effects on auditory fear, even with systemic infusions of broad-spectrum HAT or HDAC inhibitors. Some of these studies find effects on context, but not auditory fear (Korzus et al, 2004; Mahan et al, 2012; Wood et al, 2005), whereas others find effects on both context and auditory fear (Alarcon et al, 2004; Bredy and Barad, 2008; Chwang et al, 2007; Gao et al, 2010; Guan et al, 2009; Maddox et al, 2013b). Similarly, the effects of systemic HDAC3 inhibition are mixed. Systemic infusion of the HDAC3-specific inhibitor RGFP966 enhances long-term memory (including extinction memory) in multiple memory paradigms (Bieszczad et al, 2015; Malvaez et al, 2013) but fails to enhance memory consolidation for auditory fear extinction (Bowers et al, 2015). Further, chronic systemic HDAC3 inhibition with RGFP966 is not sufficient to rescue context fear memory in a mouse model of Alzheimer's Disease (Rumbaugh et al, 2015). Studies manipulating HAT or HDAC activity directly in the amygdala, on the other hand, consistently show effects on auditory fear (Maddox et al, 2013b; Maddox et al, 2013c; Monsey et al, 2011; Yeh et al, 2004), consistent with our results. Collectively, these experiments indicate that site-specific delivery of HDAC inhibitors may be necessary to reveal the role of individual HDACs in fear memory formation. To date, no other study has directly compared the effects of HDAC inhibition in the lateral versus basal subnuclei of the amygdala. Further, ours is the first study to demonstrate that disrupting HDAC activity in the amygdala can enhance context fear in addition to auditory fear, consistent with a known role of the amygdala in memory consolidation for both auditory and context fear (Kwapis et al, 2011; LeDoux, 2000).

The enhanced freezing we observed to both the context (Figures 3 and 4) and the tone (Figure 5) in AAV-HDAC3 (Y298H) animals rapidly diminished over the course of the test session. In each case, freezing expression was no longer enhanced relative to the AAV-EV control group by the end of the session. Although it is possible that HDAC3 activity disruption produces only a transient enhancement in memory formation, our past work suggests this is not the case, with HDAC3 blockade producing persistent enhancements in memory (Malvaez et al, 2013; McQuown et al, 2011a). A more plausible explanation is that the continued inhibition of HDAC3 activity during the test session contributes to enhanced extinction, resulting in reduced freezing by the end of the test session. It is currently not clear whether this within-session extinction also persists between sessions, but this would be worthy of future investigation. Consistent with this suggestion, previous research has shown that inhibition of Class I HDACs can enhance fear extinction memory (Bahari-Javan et al, 2012; Bredy and Barad, 2008; Graff et al, 2014; Lattal et al, 2007; Stafford et al, 2012; Whittle et al, 2013). Although systemic pharmacological HDAC3 inhibition fails to enhance cued fear extinction (Bowers et al, 2015), site-specific HDAC3 inhibition restricted to the hippocampus or amygdala might reveal an important role of HDAC3 in fear extinction memory. Importantly, the rapid reduction in freezing we observed during testing, along with the low levels of freezing observed during acquisition and baseline rules out the possibility that the presence of AAV-HDAC3(Y298H) drives a nonspecific increase in freezing or disrupted movement during the test session.

Our results also reveal an interesting temporal dynamic between learning-induced changes in gene expression and reduced HDAC3 occupancy. Specifically, we found that fear conditioning drove increased cFos and Nr4a2 mRNA $30 \mathrm{~min}$ after learning, whereas HDAC3 occupancy was reduced at the promoter regions of these genes only at $60 \mathrm{~min}$ post acquisition (Figure 1). One might expect changes in HDAC3 occupancy to coincide with or even precede gene expression changes after learning, as HDAC3 negatively regulates transcription. As these genes are upregulated before HDAC3 occupancy is reduced, however, it seems that learning can drive changes in gene expression even before HDAC3 is removed. One possibility is that HDAC3 activity is rapidly reduced by the learning event, possibly through reduced phosphorylation (Zhang et al, 2005), followed by the slower physical removal of HDAC3 from chromatin. This would allow for both initial, fast-onset transcription immediately after learning and the slower onset of other genes expressed later in the consolidation period. Whether learning induces this type of rapid alteration in HDAC3 activity is currently unclear but warrants further investigation.

In summary, we found that HDAC3 activity is a key negative regulator of fear memory formation in both the hippocampus and amygdala. Context fear is regulated by HDAC3 deacetylase activity in both the dorsal hippocampus and basal nucleus of the amygdala, whereas auditory CS fear 
was regulated by $\mathrm{HDAC} 3$ activity in the lateral nucleus of the amygdala. These findings confirm that the deacetylase activity of HDAC3 negatively regulates aversive memory in addition to its known role in incidental (McQuown et al, 2011a) and reward (Bieszczad et al, 2015; Malvaez et al, 2013; Rogge et al, 2013) memory.

\section{FUNDING AND DISCLOSURE}

This work was supported by the National Institute of Mental Health (NIMH) and National Institute on Aging (NIA) grants (MH101491, AG051807, AG050787) to MAW and National Institute on Aging (NIA) grants (T32 AG000096-31 and F32 AG052303) to JLK. The remaining authors declare no conflict of interest.

\section{ACKNOWLEDGMENTS}

We give special thanks to Dr Thekla Hemstedt for her help in preparing figures for publication.

\section{REFERENCES}

Alarcon JM, Malleret G, Touzani K, Vronskaya S, Ishii S, Kandel ER et al (2004). Chromatin acetylation, memory, and LTP are impaired in CBP+/- mice: a model for the cognitive deficit in Rubinstein-Taybi syndrome and its amelioration. Neuron 42: 947-959.

Alberini CM (2009). Transcription factors in long-term memory and synaptic plasticity. Physiol Rev 89: 121-145.

Allis CD, Jenuwein T, Reinberg D, Caparros ML (2007). Epigenetics, 1 edn. Cold Spring Harbor Laboratory Press: New York, NY, USA.

Amorapanth P, LeDoux JE, Nader K (2000). Different lateral amygdala outputs mediate reactions and actions elicited by a fear-arousing stimulus. Nat Neurosci 3: 74-79.

Bahari-Javan S, Maddalena A, Kerimoglu C, Wittnam J, Held T, Bahr $\mathrm{M}$ et al (2012). HDAC1 regulates fear extinction in mice. J Neurosci 32: 5062-5073.

Barrett RM, Malvaez M, Kramar E, Matheos DP, Arrizon A, Cabrera SM et al (2011). Hippocampal focal knockout of CBP affects specific histone modifications, long-term potentiation, and long-term memory. Neuropsychopharmacology 36: 1545-1556.

Barrett RM, Wood MA (2008). Beyond transcription factors: the role of chromatin modifying enzymes in regulating transcription required for memory. Learn Mem 15: 460-467.

Bieszczad KM, Bechay K, Rusche JR, Jacques V, Kudugunti S, Miao W et al (2015). Histone deacetylase inhibition via RGFP966 releases the brakes on sensory cortical plasticity and the specificity of memory formation. J Neurosci 35: 13124-13132.

Bowers ME, Xia B, Carreiro S, Ressler KJ (2015). The Class I HDAC inhibitor RGFP963 enhances consolidation of cued fear extinction. Learn Mem 22: 225-231.

Bredy TW, Barad M (2008). The histone deacetylase inhibitor valproic acid enhances acquisition, extinction, and reconsolidation of conditioned fear. Learn Mem 15: 39-45.

Canteras NS, Swanson LW (1992). Projections of the ventral subiculum to the amygdala, septum, and hypothalamus: a PHAL anterograde tract-tracing study in the rat. J Comp Neurol 324: $180-194$

Chwang WB, Arthur JS, Schumacher A, Sweatt JD (2007). The nuclear kinase mitogen- and stress-activated protein kinase 1 regulates hippocampal chromatin remodeling in memory formation. J Neurosci 27: 12732-12742.
Conkright MD, Guzman E, Flechner L, Su AI, Hogenesch JB, Montminy M (2003). Genome-wide analysis of CREB target genes reveals a core promoter requirement for cAMP responsiveness. Mol Cell 11: 1101-1108.

Gao J, Wang WY, Mao YW, Graff J, Guan JS, Pan L et al (2010). A novel pathway regulates memory and plasticity via SIRT1 and miR-134. Nature 466: 1105-1109.

Goosens KA, Maren S (2001). Contextual and auditory fear conditioning are mediated by the lateral, basal, and central amygdaloid nuclei in rats. Learn Mem 8: 148-155.

Graff J, Joseph NF, Horn ME, Samiei A, Meng J, Seo J et al (2014). Epigenetic priming of memory updating during reconsolidation to attenuate remote fear memories. Cell 156: 261-276.

Guan JS, Haggarty SJ, Giacometti E, Dannenberg JH, Joseph N, Gao J et al (2009). HDAC2 negatively regulates memory formation and synaptic plasticity. Nature 459: 55-60.

Guenther MG, Barak O, Lazar MA (2001). The SMRT and N-CoR corepressors are activating cofactors for histone deacetylase 3. Mol Cell Biol 21: 6091-6101.

Guenther MG, Lane WS, Fischle W, Verdin E, Lazar MA, Shiekhattar R (2000). A core SMRT corepressor complex containing HDAC3 and TBL1, a WD40-repeat protein linked to deafness. Genes Dev 14: 1048-1057.

Jarome TJ, Thomas JS, Lubin FD (2014). The epigenetic basis of memory formation and storage. Prog Mol Biol Transl Sci 128: 1-27.

Korzus E, Rosenfeld MG, Mayford M (2004). CBP histone acetyltransferase activity is a critical component of memory consolidation. Neuron 42: 961-972.

Kubik S, Miyashita T, Guzowski JF (2007). Using immediate-early genes to map hippocampal subregional functions. Learn Mem 14: $758-770$

Kwapis JL, Jarome TJ, Schiff JC, Helmstetter FJ (2011). Memory consolidation in both trace and delay fear conditioning is disrupted by intra-amygdala infusion of the protein synthesis inhibitor anisomycin. Learn Mem 18: 728-732.

Kwapis JL, Wood MA (2014). Epigenetic mechanisms in fear conditioning: implications for treating post-traumatic stress disorder. Trends Neurosci 37: 706-720.

Lahm A, Paolini C, Pallaoro M, Nardi MC, Jones P, Neddermann P et al (2007). Unraveling the hidden catalytic activity of vertebrate class IIa histone deacetylases. Proc Natl Acad Sci USA 104: 17335-17340.

Lattal KM, Barrett RM, Wood MA (2007). Systemic or intrahippocampal delivery of histone deacetylase inhibitors facilitates fear extinction. Behav Neurosci 121: 1125-1131.

LeDoux JE (2000). Emotion circuits in the brain. Annu Rev Neurosci 23: $155-184$.

Lehner M, Wislowska-Stanek A, Taracha E, Maciejak P, Szyndler J, Skorzewska A et al (2009). The expression of c-Fos and colocalisation of c-Fos and glucocorticoid receptors in brain structures of low and high anxiety rats subjected to extinction trials and re-learning of a conditioned fear response. Neurobiol Learn Mem 92: 535-543.

Levenson JM, O'Riordan KJ, Brown KD, Trinh MA, Molfese DL, Sweatt JD (2004). Regulation of histone acetylation during memory formation in the hippocampus. J Biol Chem 279: 40545-40559.

Lopez AJ, Kramar E, Matheos DP, White AO, Kwapis J, Vogel-Ciernia A et al (2016). Promoter-specific effects of DREADD modulation on hippocampal synaptic plasticity and memory formation. J Neurosci 36: 3588-3599.

Maddox SA, Schafe GE, Ressler KJ (2013a). Exploring epigenetic regulation of fear memory and biomarkers associated with posttraumatic stress disorder. Front Psychiatry 4: 62.

Maddox SA, Watts CS, Doyere V, Schafe GE (2013b). A naturallyoccurring histone acetyltransferase inhibitor derived from Garcinia indica impairs newly acquired and reactivated fear memories. PLoS One 8: e54463. 
Maddox SA, Watts CS, Schafe GE (2013c). p300/CBP histone acetyltransferase activity is required for newly acquired and reactivated fear memories in the lateral amygdala. Learn Mem 20: 109-119.

Mahan AL, Mou L, Shah N, Hu JH, Worley PF, Ressler KJ (2012). Epigenetic modulation of Homerla transcription regulation in amygdala and hippocampus with pavlovian fear conditioning. J Neurosci 32: 4651-4659.

Malvaez M, McQuown SC, Rogge GA, Astarabadi M, Jacques V, Carreiro $S$ et al (2013). HDAC3-selective inhibitor enhances extinction of cocaine-seeking behavior in a persistent manner. Proc Natl Acad Sci USA 110: 2647-2652.

McNulty SE, Barrett RM, Vogel-Ciernia A, Malvaez M, Hernandez N, Davatolhagh MF et al (2012). Differential roles for Nr4a1 and Nr4a2 in object location vs. object recognition long-term memory. Learn Mem 19: 588-592.

McQuown SC, Barrett RM, Matheos DP, Post RJ, Rogge GA, Alenghat $\mathrm{T}$ et al (2011a). HDAC3 is a critical negative regulator of long-term memory formation. J Neurosci 31: 764-774.

McQuown SC, Wood MA (2011b). HDAC3 and the molecular brake pad hypothesis. Neurobiol Learn Mem 96: 27-34.

Milanovic S, Radulovic J, Laban O, Stiedl O, Henn F, Spiess J (1998). Production of the Fos protein after contextual fear conditioning of C57BL/6 N mice. Brain Res 784: 37-47.

Miller CA, Campbell SL, Sweatt JD (2008). DNA methylation and histone acetylation work in concert to regulate memory formation and synaptic plasticity. Neurobiol Learn Mem 89: 599-603.

Monsey MS, Ota KT, Akingbade IF, Hong ES, Schafe GE (2011). Epigenetic alterations are critical for fear memory consolidation and synaptic plasticity in the lateral amygdala. PLoS One 6: e19958.

Rogge GA, Singh H, Dang R, Wood MA (2013). HDAC3 is a negative regulator of cocaine-context-associated memory formation. J Neurosci 33: 6623-6632.

Rumbaugh G, Sillivan SE, Ozkan ED, Rojas CS, Hubbs CR, Aceti M et al (2015). Pharmacological selectivity within Class I histone deacetylases predicts effects on synaptic function and memory rescue. Neuropsychopharmacology 40: 2307-2316.

Sando R 3rd, Gounko N, Pieraut S, Liao L, Yates J 3rd, Maximov A (2012). HDAC4 governs a transcriptional program essential for synaptic plasticity and memory. Cell 151: 821-834.
Schafe GE, Doyere V, LeDoux JE (2005). Tracking the fear engram: the lateral amygdala is an essential locus of fear memory storage. J Neurosci 25: 10010-10014.

Stafford JM, Raybuck JD, Ryabinin AE, Lattal KM (2012). Increasing histone acetylation in the hippocampus-infralimbic network enhances fear extinction. Biol Psychiatry 72: 25-33.

Sun Z, Feng D, Fang B, Mullican SE, You SH, Lim HW et al (2013). Deacetylase-independent function of HDAC3 in transcription and metabolism requires nuclear receptor corepressor. Mol Cell 52: 769-782.

Vecsey CG, Hawk JD, Lattal KM, Stein JM, Fabian SA, Attner MA et al (2007). Histone deacetylase inhibitors enhance memory and synaptic plasticity via CREB:CBP-dependent transcriptional activation. J Neurosci 27: 6128-6140.

Vogel-Ciernia A, Wood MA (2012). Molecular brake pad hypothesis: pulling off the brakes for emotional memory. Rev Neurosci 23: 607-626.

White AO, Kramar EA, Lopez AJ, Kwapis JL, Doan J, Saldana D et al (2016). BDNF rescues BAF53b-dependent synaptic plasticity and cocaine-associated memory in the nucleus accumbens. Nat Commun 7: 11725.

Whittle N, Schmuckermair C, Gunduz Cinar O, Hauschild M, Ferraguti F, Holmes A et al (2013). Deep brain stimulation, histone deacetylase inhibitors and glutamatergic drugs rescue resistance to fear extinction in a genetic mouse model. Neuropharmacology 64: 414-423.

Wood MA, Kaplan MP, Park A, Blanchard EJ, Oliveira AM, Lombardi TL et al (2005). Transgenic mice expressing a truncated form of CREB-binding protein (CBP) exhibit deficits in hippocampal synaptic plasticity and memory storage. Learn Mem 12: 111-119.

Yaniv D, Desmedt A, Jaffard R, Richter-Levin G (2004). The amygdala and appraisal processes: stimulus and response complexity as an organizing factor. Brain Res Brain Res Rev 44: 179-186.

Yeh S, Lin C, Gean P (2004). Acetylation of nuclear factor- $\kappa B$ in rat amygdala improves long-term but not short-term retention of fear memory. Mol Pharmacol 65: 1286-1292.

Zhang X, Ozawa Y, Lee H, Wen YD, Tan TH, Wadzinski BE et al (2005). Histone deacetylase 3 (HDAC3) activity is regulated by interaction with protein serine/threonine phosphatase 4 . Genes Dev 19: 827-839.

Supplementary Information accompanies the paper on the Neuropsychopharmacology website (http://www.nature.com/npp) 